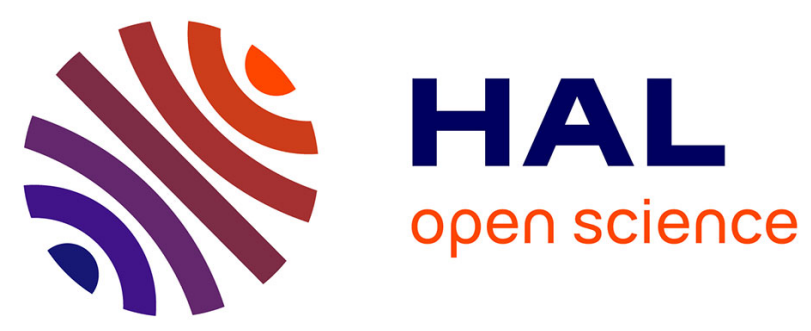

\title{
Influence of intramolecular charge coupling on intermolecular interactions of polycarboxybetaines in aqueous solution and in polyelectrolyte multilayers
}

Thomas Schimmel, Jörg Bohrisch, Dan F Anghel, Julian Oberdisse, Regine von Klitzing

\section{To cite this version:}

Thomas Schimmel, Jörg Bohrisch, Dan F Anghel, Julian Oberdisse, Regine von Klitzing. Influence of intramolecular charge coupling on intermolecular interactions of polycarboxybetaines in aqueous solution and in polyelectrolyte multilayers. Molecular Physics, 2021, 119 (15-16), pp.6722-6733. 10.1080/00268976.2021.1936676 . hal-03384120

\author{
HAL Id: hal-03384120 \\ https://hal.science/hal-03384120
}

Submitted on 18 Oct 2021

HAL is a multi-disciplinary open access archive for the deposit and dissemination of scientific research documents, whether they are published or not. The documents may come from teaching and research institutions in France or abroad, or from public or private research centers.
L'archive ouverte pluridisciplinaire HAL, est destinée au dépôt et à la diffusion de documents scientifiques de niveau recherche, publiés ou non, émanant des établissements d'enseignement et de recherche français ou étrangers, des laboratoires publics ou privés. 


\title{
Influence of intramolecuar charge coupling on intermolecular interactions of polycarboxybetaines in aqueous solution and in polyelectrolyte multilayers
}

\author{
Thomas Schimmel ${ }^{a, b}$, Jörg Bohrisch ${ }^{a}$, Dan F. Anghel ${ }^{c}$, \\ Julian Oberdisse $^{d, e *}$ and Regine von Klitzing $f *$
}

${ }^{a}$ Fraunhofer-Institut für Angewandte Polymerforschung, D-14476 Golm

${ }^{b}$ Present address: Evonik (China) Co., Ltd. 55 Chundong Road, Xinzhuang Industry Park, CHN-Shanghai 201108

c "Ilie Murgulescu" Institute of Physical Chemistry, Laboratory of Colloids, Spl. Independentei 202, RO - 77208 Bucharest

${ }^{d}$ Laboratoire Charles Coulomb (L2C), Université de Montpellier, CNRS, F34095 Montpellier

${ }^{e}$ Laboratoire Léon Brillouin (LLB), CEA/CNRS, CEA Saclay, F-91190 Gifsur-Yvette

${ }^{f}$ Soft Matter at Interfaces, Department of Physics, Technische Universität Darmstadt, Hochschulstraße 8, D-64289 Darmstadt

\begin{abstract}
The present paper deals with the correlation between intramolecular and intermolecular interactions of polycarboxybetaines. The degree of coupling between the opposite charges within the polycarboxybetaine molecules was varied by spacers of different length and by
\end{abstract}

*Corresponding authors: Julian Oberdisse (julian.oberdisse@umontpellier.fr) and Regine von Klitzing (klitzing@smi.tu-darmstadt.de) 
the substitution of additional alkyl chains at the quaternary nitrogen atom. In order to check intermolecular interactions between polycarboxybetaines, SANS measurements were carried out at aqueous polycarboxybetaines solutions. For getting information about the interaction with an oppositely charged polyelectrolyte, multilayers were formed by alternating adsorption of polystyrene sulfonate (PSS) and polycarboxybetaines from aqueous solutions. The occurrence of a structure peak in SANS spectra and the ability to form polyelectrolyte multilayers provide an indicator for the polyelectrolyte character of some of the studied polycarboxybetaines. Not only the charge but also the hydrophobicity of the polycarboxybetaines has a pronounced effect on the chain conformation and therefore on the thickness of the polyelectrolyte multilayers. The results show that small differences in molecular architecture lead to pronounced differences in intermolecular interactions.

\section{Keywords}

Polycarboxybetaines, intermolecular interactions, intramolecular interactions, polyelectrolyte multilayer, charge reversal, polymer charge density, ionic strength.

\section{Introduction}

Polyelectrolytes in aqueous media are of interest for many technical applications and in basic research. Polyelectrolytes can be used as electrostatic and/or sterical stabilizer for colloidal dispersions. On the other hand they act as destabilizer if they induce depletion, and they can be salted out easily. Usually, they are water soluble and non-toxic which makes them interesting for many chemical formulations. For theoretical considerations they are attractive since they show properties of electrolytes and polymers and both fields should be combined to describe their complex behavior. For several decades the conformation and ordering of polyelectrolyte chains in aqueous 
media has been investigated by scattering $[1,2,3,4,5]$ and described by theoretical calculations $[6,7,8,9,10]$. Synthetic polyelectrolytes can be used as model systems for natural polymers like DNA or proteins. Especially the latter ones are very complex, since they have hydrophobic and hydrophilic areas and many of them show zwitterionic behavior. In the case of zwitterionic molecules the number of positive and negative charges decides about the net charge of the molecule. For the stabilisation of proteins betaines are of interest. They are zwitterionic with a strong electric dipole, and the positive charge (nitrogen) carries a hydrophobic cap. Hence, they are hydrophobic molecules with a strong electric dipole. As a consequence they favor adsorption neither at hydrophobic surfaces (or molecules) due to their large amount of water around the dipole nor at hydrophilic surfaces (or molecules) due to their charges [11]. Therefore betaines acts similar as Trimethylaminoxid (TMAO) - a well-known osmolyte - and stabilize proteins by preventing them from defolding. The question arises how important the charge coupling is for the interaction with other molecules. If for instance one charge is screened and the charge coupling is interrupted the opposite charge might dominate the intermolecular interaction, like for polycarboxybetaines studied in the present paper. They are zwitterionic with a permanent positively charged nitrogen atom and a negative charge caused by an acrylic acid group which is protonated below a $\mathrm{pH}$ of about 4 and deprotonated at intermediate and high $\mathrm{pH}$ values. In the latter $\mathrm{pH}$ regime the net charge of the polycarboxybetaines is zero, and it is positive in the acidic medium. Capillary electrophoresis experiments indicates that the charges within one poly(carboxybetaine) neutralize each other if a) opposite charges have a short distance and if b) none of the charges are screened [12]. The question arises if this intramolecular charge interaction is also reflected in intermolecular interactions. In the present paper the intermolecular interaction between polycarboxybetaines is studied via 1) the ability for structure formation in the aqueous bulk solution and 2) the ability to form multilayers with an oppositely charged polyelectrolyte. These studies are also of interest 
for technical applications as for thickening of paintings and surface coatings. The structure formation in the bulk was investigated by small angle neutron scattering (SANS). In general, scattering spectra of aqueous solutions of polyelectrolytes containing one type of charge show a broad intensity peak $\left(q_{\max }=2 \pi /\right.$ correlation length $)$ which reflects the interactions between the chains. In the dilute regime $\left(c<c^{*}\right)$ the peak position scales with the polyelectrolyte concentration $c$ as $q_{\max } \propto c^{1 / 3}$, which is typical for an isotropic distribution of molecules in three dimensions and in the semi-dilute regime $\left(\mathrm{c}>\mathrm{c}^{*}\right)$ it is $q_{\max } \propto c^{1 / 2}$ which is related to the overlap of polyelectrolyte chains and the formation of a transient network [13]. With increasing ionic strength or decreasing charge density the peak vanishes which indicates that the peak is due to electrostatic repulsion between the polyelectrolyte chains $[14]$.

In the present paper different classes of polycarboxybetaines are studied. The distance between both charges (parameter $\mathrm{k}$ ) is changed by the number of $\mathrm{CH}_{2}$ groups and the screening of the charges (parameter $\mathrm{R}$ ) is varied by the length of alkyl side chains at the nitrogen. In these experiments an eventual occurrence of a structure peak would indicate the polyelectrolyte character of the polycarboxybetaines. The polyelectrolyte multilayers are formed by the layer-by-layer technique suggested by Decher and Coworkers in the early 1990's [15]. Usually, the multilayers are built-up by alternate adsorption of polycations and polyanions from aqueous solutions. The thickness of the multilayer increases with increasing ionic strength of the dipping polyelectrolyte solutions. Due to the charge screening along the polyelectrolyte chain the chains adsorb in a more coiled conformation than at low ionic strength. Beside this segment-segment interaction also the segment-interface interaction is screened which has an opposite effect on the thickness. Hence, there should be a maximum in multilayer thickness where the repulsion of likely charged polyelectrolyte segments is partially screened, but not overcompensated by the screening of the attraction between oppositely charged segments. If the polyelectrolyte character of the polycarboxybetaines is pronounced 

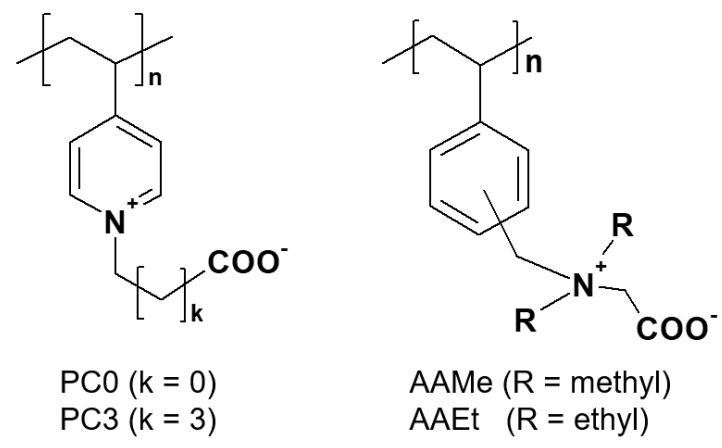

Figure 1: Poly(carboxybetaines) with different spacers between the charges and different lengths of side chains at the quaternary nitrogen.

they should build up multilayers with an oppositely charged polyelectrolyte and the described effect of the ionic strength should be detectable.

\section{Experimental section}

\section{$2.1 \quad$ Materials}

The synthesis of the polycarboxybetaines is described elsewhere [12]. The structure and the molecular parameters are shown in fig. 1 and in table 1. Four different polycarboxybetaines have been studied: Pyridiniocarboxylates (PC) with two different spacer lengths between both charges $\mathrm{k}=0$ (PC0) and $\mathrm{k}=3$ (PC3) and two ammonioacetates (AA) where the charges are separated by one $\mathrm{CH}_{2}$ group and the quaternary nitrogen carries methyl (AAMe) or ethyl side chains (AAEt). While the nitrogen in $\mathrm{PC} 0$ and $\mathrm{PC} 3$ has a $\mathrm{sp}^{2}$ hybridation the nitrogen in AAMe and AAEt has a $\mathrm{sp}^{3}$ hybridation. Therefore the positive charge in $\mathrm{PC} 0$ and $\mathrm{PC} 3$ is rather dislocated while it is located in AAMe and AAEt. 


\begin{tabular}{|c|c|c|c|c|c|c|c|c|}
\hline $\begin{array}{c}\text { Sample } \\
\text { name }\end{array}$ & $\begin{array}{c}\mathrm{k} \text {, or } \\
\text { side group }\end{array}$ & $\begin{array}{c}\mathrm{M}_{\text {mon }} \\
\mathrm{g} / \mathrm{mol}\end{array}$ & $\begin{array}{c}\mathrm{M}_{n} \\
\mathrm{~g} / \mathrm{mol}\end{array}$ & $\begin{array}{c}\mathrm{M}_{w} \\
\mathrm{~g} / \mathrm{mol}\end{array}$ & $\mathrm{PI}$ & $\mathrm{N}$ & $\Phi$ & $\begin{array}{c}\Delta \rho \\
\left(10^{10} \mathrm{~cm}^{-2}\right)\end{array}$ \\
\hline PC0 & $\mathrm{k}=0$ & 163 & 17900 & 27200 & 1.52 & 167 & $1.8 \%$ & 4.66 \\
\hline PC3 & $\mathrm{k}=3$ & 205 & 18800 & 23880 & 1.27 & 116 & $2.3 \%$ & 5.09 \\
\hline AAMe & $\mathrm{R}=$ methyl & 219 & 13100 & 16900 & 1.29 & 77 & $2.3 \%$ & 5.20 \\
\hline AAEt & $\mathrm{R}=$ ethyl & 247 & 12900 & 16640 & 1.29 & 67 & $2.7 \%$ & 5.37 \\
\hline
\end{tabular}

Table 1: Sample composition for pyridiniocarboxylates $(P C)$ and ammonioacetate (AA), monomer molecular mass $M_{m o n}$, polymer number and weight average mass $\left(M_{n}\right.$ and $\left.M_{w}\right)$, polydispersity index $P I$, degree of polymerisation $N$ $\left(M_{w} / M_{\text {mon }}\right)$ polymer volume fraction $\Phi$ of most concentrated sample in $D_{2} \mathrm{O}$ (corresponds to 0.1 monomol/L), and coherent neutron scattering contrast $\Delta \rho$ in $D_{2} O$.

\subsection{Multilayer preparation}

Branched polyethylene imine (PEI) and poly(styrene sulfonate) sodiumsalt (PSS) were obtained from Aldrich (Steinheim, Germany). The molecular weight of PEI was $750.000 \mathrm{~g} / \mathrm{mol}$ and 70.000 in the case of PSS. The polymer solutions contained $10^{-2}$ monomol/L (concentration of monomer units) of the respective polyelectrolyte in Milli-Q-water. The ionic strength of the respective aqueous polymer solution was adjusted by $\mathrm{NaCl}$ and the $\mathrm{pH}$ of the polycarboxybetaine solutions was adjusted by $\mathrm{HCl}$. Unless stated otherwise the $\mathrm{pH}$ of the PSS solutions was not adjusted and was about 4.6. In all solutions the $\mathrm{NaCl}$ concentration was higher than the $\mathrm{H}_{3} \mathrm{O}^{+}$concentration. With exception of $\mathrm{pH} 1.5$ and a $\mathrm{NaCl}$ concentration of $0.1 \mathrm{~mol} / \mathrm{l}$, the variation of the ionic strength caused by the $\mathrm{pH}$ adjustment was less than $10 \%$. The Silicon wafers were provided by Wacker Siltronic AG, Burghausen (Germany) and cleaned for $30 \mathrm{~min}$ in $1: 1 \mathrm{H}_{2} \mathrm{O}_{2} / \mathrm{H}_{2} \mathrm{SO}_{4}$ mixture. After that they were coated with a PEI layer. Then, PSS and polycarboxybetaine were deposited consecutively via the layer-by-layer technique on the silicon wafers by immersion for 20 min into the respective aqueous polymer solutions and 
by rinsing with Milli-Q-water after each deposition step. A complete dipping cycle in the following concerns to the sequence PSS/ water/ polycarboxybetaine/ water. The outer layer was always the polycarboxybetaine. The films were dried in an air stream after completion of the multilayer assembly. For the multilayer formation the polycarboxybetaines either AAMe or AAEt were used.

\subsection{Apparatus and measurement procedure}

\subsubsection{Small angle neutron scattering (SANS)}

The SANS measurements have been carried out on beamline PACE at Laboratoire Léon Brillouin (CEA/CNRS) in Saclay. PACE was a traditional small angle instrument with pinhole collimation and mechanical velocity selector. Two configurations were used, with fixed wavelength $(6 \AA)$ and sample-todetector distance $1.0 \mathrm{~m}$ and $4.6 \mathrm{~m}$, giving a q-range from $7 \times 10^{-3} \AA^{-1}$ to 0.32 $\AA^{-1}$. All polymer samples were measured in $\mathrm{D}_{2} \mathrm{O}$ in $2 \mathrm{~mm}$ Hellma-cuvettes. Empty cell scattering was subtracted and $\mathrm{H}_{2} \mathrm{O}$ incoherent scattering was used to correct for detector imperfections. Incoherent scattering from the samples comes mainly from the solvent, due to the low concentrations of hydrogenated material used. We have thus subtracted a constant but individual value (given by $\mathrm{D}_{2} \mathrm{O}$ scattering, $\approx 0.05 \mathrm{~cm}^{-1}$ ) from all spectra. Finally, the scattered intensity was converted in absolute units $\left(\mathrm{cm}^{-1}\right)$ by an independent measurement of the direct beam intensity. The neutron scattering length densities of the compounds have been calculated from the contributions of each atom in a repeat unit, divided by its volume estimated from a density of $1 \mathrm{~g} / \mathrm{cm}^{3}$. Counterion contributions are negligible, and moreover difficult to attribute spatially due to their mobility. They have thus not been

included in the scattering contrast with respect to the solvent given in table 1. 


\subsubsection{Ellipsometry}

The polyelectrolyte multilayers at the solid interfaces were characterized by ellipsometry. By this method one gets information about the thickness and the refractive index of the coating. For a detailed description of ellipsometry the reader is referred to $[16,17]$.

For thin films $(\mathrm{d}<100 \AA)$ the variation of the polarizer angle is very minor. Only the analyzer angle changes significantly, and therefore only one parameter (thickness or refractive index) of the coating can be determined. In the present study the refractive index is taken from a thick film $(\mathrm{n} \approx 1.5)$ under the assumption that the refractive index is the same for thin films. A Multiscope from Optrel (Berlin, Germany) has been used for the experiments presented in the following.

Each type of film has been prepared at least twice. The thickness has been measured at several positions on the wafer by ellipsometry (foot print: around $1 \times 3 \mathrm{~mm})$. The presented thickness data are average values and the error bars are smaller than the symbol size.

\section{Results and discussion}

\subsection{Aqueous bulk solution (SANS)}

Concentrations from 0.1 down to $3.125 \times 10^{-3}$ monomol/L of polycarboxybetaines have been measured by SANS, obtained by successively dividing the concentration by two. Fig. 2 shows the reduced SANS intensities I(q)/ $\Phi$ of the four samples at different concentrations, in $\mathrm{D}_{2} \mathrm{O}$ at $\mathrm{pH} 2$ adjusted by addition of $\mathrm{HCl}$. All SANS samples have been prepared without added salt for reasons of colloidal stability. Due to the presence of $\mathrm{HCl}$, and counterions introduced by each repeat unit, the Debye screening length already rather

low, typically $10 \AA$ for $0.1 \mathrm{~mol} / \mathrm{L}$ solutions, i.e. it corresponds to a high ionic strength with considerably electrostatic screening.

All intensities $\mathrm{I}(\mathrm{q})$ have been divided by their corresponding polymer volume 

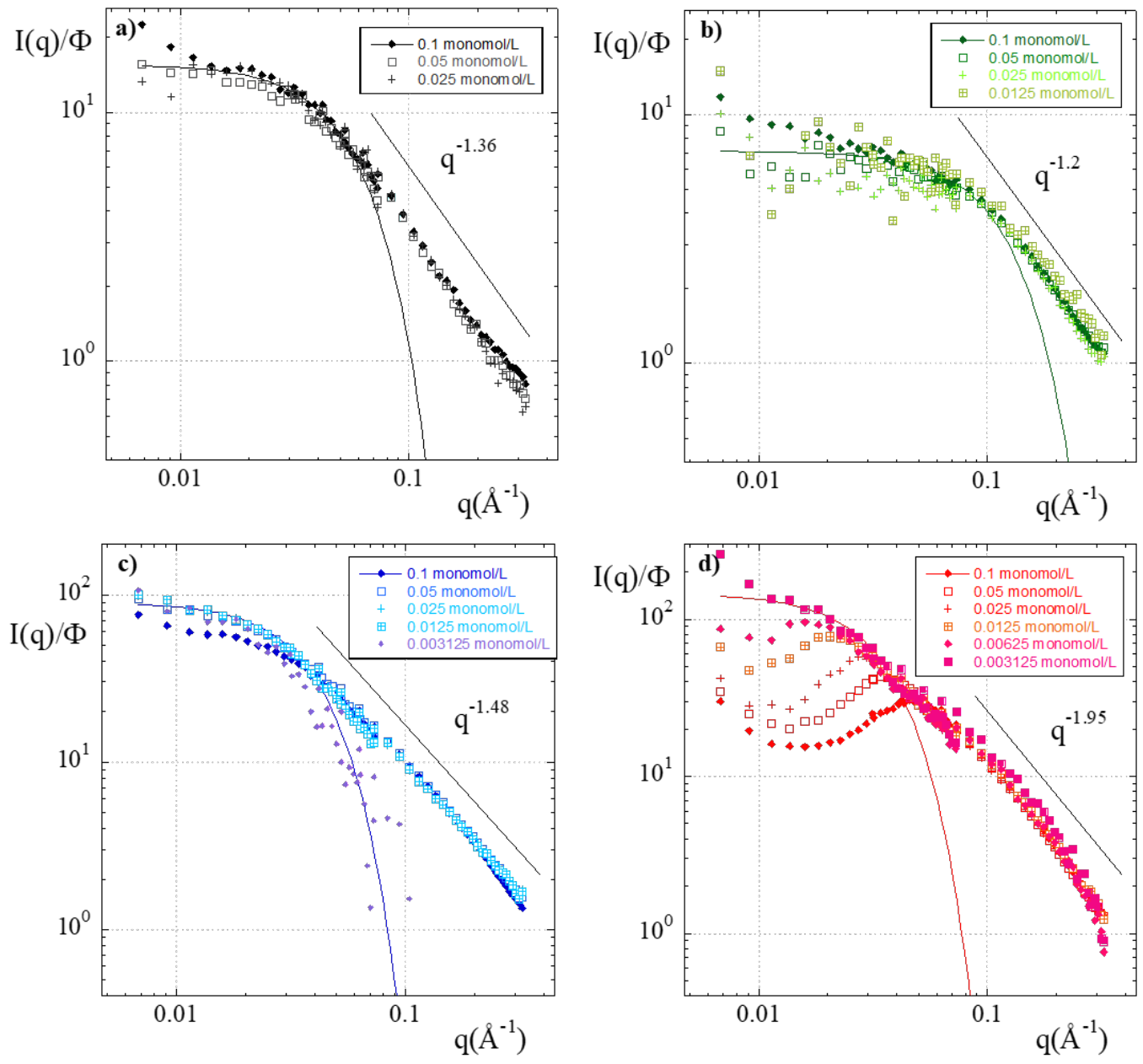

Figure 2: Reduced SANS intensities for a concentration series starting from 0.1 monomol/L of polycarboxybetaines a) PCO b) PC3 c) AAMe d) AAEt. The lines correspond to fits of the low q-regime and to the high-scaling law, respectively. 
fraction $\Phi$, obtained by assuming a polymer density of $1 \mathrm{~g} / \mathrm{cm}^{3}$ again, which is found to be in the percent range as given in table 1. At very low concentrations, the scattered intensity becomes very weak, and some curves are not shown because of the insufficient statistics. The intensities of samples PC0, PC3, and AAMe are monotonously decreasing functions with increasing wave vector, whereas the polymer AAEt displays a strong structure factor peak. This indicates that the molecules PC0, PC3, and AAMe carry no or little net electrostatic charge, which could be due to an intramolecular neutralization mechanism. To address this, one has to consider the intramolecular charge coupling within the polycarboxybetaines. In general, at $\mathrm{pH} 2$ the carboxyl groups are protonated and therefore uncharged: the $\mathrm{pK}_{a}$ is 5.1 for poly(acrylic acid), and 4.7 for acrylic acid. Therefore the total charge of the side group is determined by the positive ammonium charge. However, if this positive charge comes to close vicinity of the carboxyl group, it competes with the $\mathrm{H}^{+}$for the charge compensation of the carboxyl group, and the carboxyl group might be deprotonated even at low $\mathrm{pH}$, leading to an overall neutral side group. If the distance to the quaternary nitrogen is small, the carboxyl group might be dissociated, and the total molecule becomes neutral, i.e. exhibit intramolecular charge compensation even at low pH. This mechanism can be described as a lower virtual $\mathrm{pK}_{a}$ value. With increasing distance or steric hinderance between the nitrogen and the carboxyl group, the probability for protonation of the carboxyl group increases, corresponding to an increase of the virtual $\mathrm{pK}_{a}$ value. These arguments are in line with electrophoretic mobility measurements of the polycarboxybetaines, which have shown that the net charge of compound PC3 is higher than the one of PC0, and the electrophoretic mobility of AAEt is higher than the one of AAMe [12]. However, as none of the three compounds PC0, PC3 and AAMe displays a structure factor peak, the total charge can be assumed to be too small to overcome electrostatic screening, even for molecule PC3. Also, going from $\mathrm{PC} 0$ to $\mathrm{PC} 3$, the longer spacer between $\mathrm{N}^{+}$and the carboxyl group may induce interactions with neighbouring side chains, providing a counter- 


\begin{tabular}{|c|c|c|c|c|}
\hline Sample & High-q exponent $a( \pm 0.05)$ & $\mathrm{R}_{\text {blob }}(\AA)( \pm 2 \AA)$ & $\mathrm{M}_{\text {blob }}(\mathrm{g} / \mathrm{mol})$ & $\mathrm{N}_{\text {blob }}$ \\
\hline PC0 & 1.36 & 28 & 4300 & 6.3 \\
\hline PC3 & 1.20 & 13 & 1650 & 14.5 \\
\hline AAMe & 1.48 & 44 & 20075 & 0.8 \\
\hline AAEt & 1.95 & 50 & 30250 & 0.6 \\
\hline
\end{tabular}

Table 2: Exponent a of power high-q power law, apparent radius of gyration of the blob $R_{b l o b}$, apparent mass of blob $M_{b l o b}$, number of blobs per chain $N_{b l o b}$.

acting trend in favour of deprotonation of neighboured carboxyl groups and a decreasing net charge.

For ammonioacetates, the charge of the $\mathrm{sp}^{3}$ hybridized ion is more located than the $\mathrm{sp}^{2}$ hybridized ion in the pyridiniocarboxylates. Especially in compound AAEt the steric hindering by the ethyl group is pronounced which counteracts a charge compensation of neighboured carboxyl groups. Due to these reasons sample AAEt is assumed to have the highest positive net charge. This leads to a strong intermolecular repulsion between the polymers of type AAEt, which is indicated by the occurrence of a pronounced structure peak in the SANS spectra.

Coming back to the non-interacting compounds, the general shape of the intensity curves of samples PC0, PC3 and AAMe in fig. 2 can be described by two regimes: a Guinier regime at low q-range followed by a power law in the high q-range. The latter has been superimposed for illustration in the plots.

\subsubsection{High q-range}

The power law exponent is characteristic of the average local chain conformation [18]. $\mathrm{A} \mathrm{q}^{-2}$ law, e.g., is typical for a Gaussian coil, whereas a lower exponent indicates more linear chains, down to one for stiff rods. The measured value may be an average over different conformations along the same chain. The results obtained from fig. 2 are reported in table 2 . 
For the pyridiniocarboxylates, the power law remains roughly the same, indicating rather extended chains, with possibly a slight increase of the net charge (inducing chain stiffening) as one goes from PC0 (1.36) to PC3 (1.2). This agrees with the above argument of decreasing intramolecular charge compensation, which makes PC3 more charged than PC0. For the ammonioacetates, the power law exponent increases from 1.5 to almost 2 , indicating a gradual transition from on average more stretched to more coil-like chains. The ammonioacetates even reach the typical conformation of an ideal chain, of fractal dimension 2. This is surprising, as such a trend towards Gaussianity is usually associated with a decreasing repulsion between equally charged monomers, whereas the AAEt chains are certainly more charged than the AAMe chains due to reduced charge coupling by the bulkier and more hydrophobic ethyl groups. Due to an increase in hydrophobicity, AAEt chains favour a more pronounced coil-like structure in comparison to AAMe by compensating partially the internal electrostatic repulsion between monomers. There is, however, no electrostatic screening effect, and the compensation by hydrophobic interactions does not outweigh the total increase in electrostatic charge of each molecule, which leads to the repulsive peak. That means two counteracting effects of increasing charge and local hydrophobicity have to be taken into account, discussing the chain conformation. A certain hydrophobicity of polyelectrolyte chains leads to the alignment of electrostatic blobs (pearls, beads) separated by strings. This structure is called pearl-necklace structure and was described e.g. by Dobrynin, Colby and Rubinstein [9]. The scattering factor of pearl-necklace chains was calculated by Huber and coworkers $[19,20]$, but due to the absence of the corresponding signature in the present scattered intensities, it could not be applied here.

\subsubsection{Guinier regime}

In the intermediate to low-q range, below $0.1 \AA^{-1}$, there is a cross-over to the flat scattering of the Guinier regime. In some cases, usually at the highest concentrations, the intensity does not stay constant at low q, but in- 
creases slightly again towards very small q values. This indicates additional large-scale structures, the exact nature of which can not be extracted unambiguously from these data. The use of larger wavelengths (16.8 $\AA$ ) had been attempted to reach lower q, but the low flux did not allow further measurements. Ignoring possible low-q upturns, the scattering curve is typical for finite sized-objects, and the position of the cross-over can be analysed in terms of the radius of gyration $\mathrm{R}_{g}$ of these objects [21]. In the following the objects are considered as blobs with a radius of gyration $\mathrm{R}_{b l o b}$ :

$$
I=I_{0} \exp \left(-q^{2} R_{b l o b}^{2} / 3\right)
$$

The radii of gyration averaged over the different concentrations are given in table 2. In practice, they have been obtained by fitting eq. (1) to each experimental intensity, decreasing progressively the q-range until a satisfactory agreement with the data was obtained. In theory, the Guinier range should extend until (at most) $\mathrm{q}_{m} a x=1 / \mathrm{R}_{g}$. As can be seen from the Guinier functions superimposed to the data in Figure 2, it is actually still acceptable beyond this value, as often observed for polydispersed fuzzy objects. On the other hand, the av-erage of the fit functions is not always good at low q, as one can simply guess from the scattering of the data in this range. In some cases, as with the highest concentration in fig. 2a, one can even observe some attractive interactions leading to a low-q upturn, which is of course not captured by the simple Guinier model of independent blobs. All in all, however, one may note that the order of magnitude of both the mass - via the average intensity level - and the spatial extension - through the kink in the intensity - is measured in a robust manner.

\section{a) Pyridiniocarboxylates PCO and PC3}

For the pyridiniocarboxylates, $\mathrm{R}_{b l o b}$ is found to decrease strongly (from 28 $\AA$ (PC0) to $13 \AA$ (PC3)), and this is accompanied by a decrease in total intensity between Fig. 2a and b. When $\mathrm{k}$ is increased from 0 to 3, the blobs become thus considerably smaller and lighter, although the mass of both 

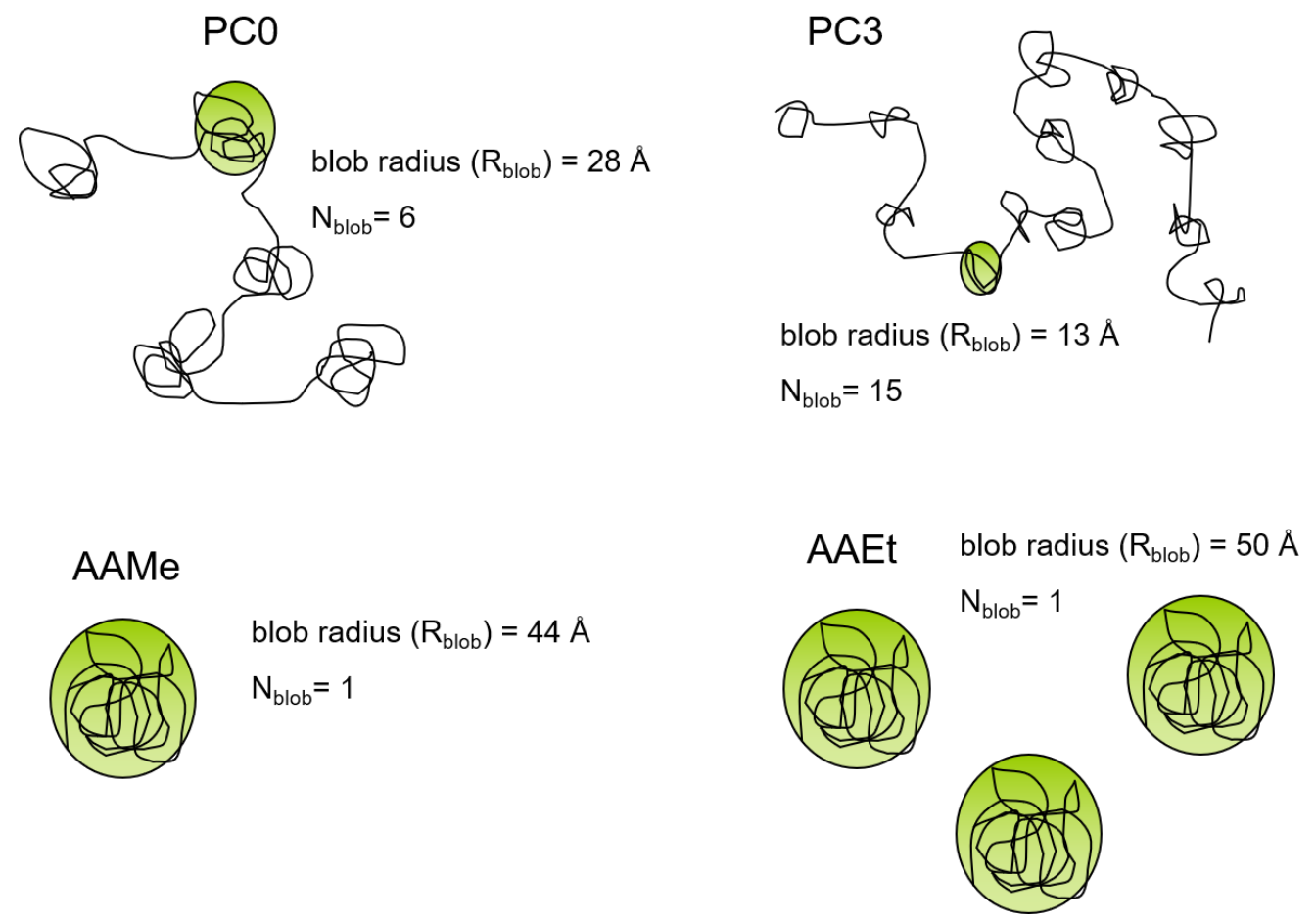

Figure 3: Schemes of the chain conformations of the four studied polycarboxybetaines deduced from SANS measurements.

carboxybetaine chains is comparable. This means that in the low q-range the SANS measurements only probe smaller correlated objects (blobs), but not the polymer as a whole. The chain is subdivided into independent units, i.e. blobs, as shown in fig. 3 .

In absence of a structure factor as observed in Figs. 2(a-c), the low angle limit of $\mathrm{I}(\mathrm{q})$ gives the apparent dry volume of the blobs $\mathrm{V}_{\text {blob }}$ :

$$
I_{0}=\Phi \Delta \rho^{2} V_{b l o b}
$$

where $\Phi$ is the total volume fraction of polymer, and $\Delta \rho$ the contrast given in table $1 . \mathrm{V}_{\text {blob }}$ can then be converted into the apparent mass $\mathrm{M}_{\text {blob }}$ which is also given in table 2. As seen qualitatively in fig. 2, the apparent mass of the blobs decreases strongly from $\mathrm{k}=0$ to $\mathrm{k}=3$. At the same time, the observed 
radius of gyration decreases. In the framework of the intramolecular charge compensation scheme outlined above, this can be understood as the result of the increased net charge from $\mathrm{PC} 0$ to $\mathrm{PC} 3$, which tends to stretch chain and organize it in smaller and more numerous blobs. By dividing the total chain mass $\mathrm{M}_{w}$ (table 1) by $\mathrm{M}_{b l o b}$, and an approximate number of blobs per chain, $\mathrm{N}_{\text {blob }}$, can be deduced. This number is found to increase from about 6 to 15. Electrostatic interactions seem to be screened on the scale of the distance between blobs, as no signature of a structure factor is found in the SANS data. Indeed, the Debye screening length has been estimated to a minimum of $10 \AA$ (corresponding to the highest polymer concentration 0.1 monomol/L), which is smaller or in the same range as the blob size. These results give strong hints for a pearl-necklace structure as illustrated in fig. 3 and mentioned already above. The fact that the the blob size decreases with increasing degree of polyelectrolyte charge was also found for polystyrene sulfonate (PSS) in water [22]. Improvement of the solvent quality by adding tetrahydrofuran (THF) had the same effect.

As a final remark on this analysis of the Guinier regime for $\mathrm{PC} 0$ and $\mathrm{PC} 3$, one may estimate the internal volume fraction of polymer within a blob from $\mathrm{R}_{b l o b}$ and the apparent mass, supposing a spherical and homogeneous spatial mass distribution in blobs. The small blobs of compound PC3 are found to be a lot denser (15\%) than for the less charged PC0 (4\%). Given the low concentrations (table 1), the blobs are thus separated in space and do not overlap. The same is not true, however, for the entire chain. Indeed, the overlap concentration of entire blobs of the same conformation as a single blob (given by the high q power law exponent a) can be estimated by applying a power law to the blob. It results that the internal concentration scales as $1 / N^{3 / a-1}$, and we find $0.3-0.4 \%$ for both PC- molecules. Blobs of entire chains would thus overlap down to the lowest concentrations, while their smaller blobs are well-separated and are probably made from a single chain. It looks like the more charged molecule tends to distribute its electrostatic charge over space by making many small blobs, which however (and 
counter-intuitively) are denser. It is possible that the structure of blobs and connections between them corresponds to a different molecular organization inside and outside blobs, and that the high-q power law in table 2 averages over both, leading to the observed decrease from 1.36 to 1.2.

\section{b) Ammonioacetates $A A M e$ and $A A E t$}

The same analysis following eqs. 1 and 2 has been applied to the ammonioacetates, and results are also provided in table 2 . Note that due to the presence of the interaction peak shown in fig. $2 \mathrm{~d}$, only the lowest concentration sample of AAEt had a Guinier regime. For both ammonioacetates, the radius of gyration and the apparent mass are much bigger than for the pyridiniocarboxylates, and within error bars, the molecules form rather large single coils, which are considered as one blob in the present picture $\left(\mathrm{R}_{\text {blob }} \approx 50 \AA\right.$, $\mathrm{N}_{b l o b}=1$ ). In case of ammonioacetates $\mathrm{R}_{b l o b}$ corresponds more or less to the radius of gyration of the entire polmyer chain. Their internal volume fraction can be estimated to $5 \%$ for both AAMe and AAEt, i.e. these blobs contain $95 \%$ of water. If one compares the internal to the highest total nominal volume fraction given in table 2 , one reaches the conclusion that blobs of compounds PC0, AAMe and AAEt are closer to contact in the suspension (because the internal volume fraction, or equivalently the overlap concentration, is only a factor of two or three from the global one), whereas the ones of the denser PC3 are more separated under these conditions. Coming back to the surprisingly similar internal concentrations of AAMe and AAEt, they might be due to the com-pensation of size and chain conformation. While the stronger high-q exponent for the more hydrophobic AAEt tends towards an increase in density, the increase in volume by a factor of two, with possibly aggregation of one or two molecules $\left(1 / \mathrm{N}_{\text {blob }}=1.7\right)$ in each blob, may lead

to a lower average density (proportional to $1 / \mathrm{N}^{3 / a-1}$, very different for the two samples) due to the fractal nature of polymer molecules."

The AAEt compound is the only one displaying a prominent scattering peak in the intensity curve, cf. fig. 2d. Such a peak is usually associated with 
a non-zero total electric charge of the chains, causing mutual repulsion and thereby inducing a liquid-like ordering of the solution, i.e. the largest possible distance (compatible with conservation of polymer mass) between objects made of single chains. Fig. 2d shows that intensities divided by the concentration clearly overlap in the intermediate q-range. This speaks in favour of identical objects which follow a dilution law. To check this we have plotted in fig. 4 the peak position $q_{\max }$ as determined from fig. 2d. With increasing AAEt polymer concentration, the peak is shifted to higher q-values. The log-log presentation shows a clear evidence for a power law, and the peak position scales with the polymer volume fraction $\Phi$ as $q_{\max } \propto \Phi^{0.38}$. The exponent 0.38 is close to $1 / 3$ shown for comparison, and it indicates a dilute regime, where the polyelectrolyte coils are not interdigitating and are isotropically diluted as solvent is added, as commonly observed for repulsively interacting polyelectrolytes in the dilute regime [13], micelles of ionic surfactants or colloidal particles [23]. The exponent 1/3 indicates a simple three-dimensional packing [24]. Such an evolution of the peak position with concentration has been known for a long time, and it has for example been discussed in the 80s for globular micelles [25]. These authors compared the prediction of the cubic model to statistical mechanical theories based on the solution of the Ornstein-Zernike integral equation for long-range electrostatic repulsion $[26,27]$. For strongly charged colloidal particles like silica particles it turned out that the scaling exponent is $1 / 3$, and that $q_{\text {max }}$ scales with the particle number density $n$ as $q_{\max }=n^{1 / 3}$ (equals sign!) [23]. Another explanation for the exponent $1 / 3$ might be the blob-controlled semi-dilute regime [22]. This can be excluded since only one blob per chain could be detected in the present study. Under the assumption that the radius of gyration of the blob and of the entire AAEt chain are similar the maximum concentration of $0.1 \mathrm{monomol} / \mathrm{L}$ is still below the overlap concentration $\mathrm{c}^{*}$.

In such systems the repulsion associated with the conservation of matter defines a typical length scale between objects. In the case of strong repulsion, the pair correlation function is peaked at this distance, and the same holds 


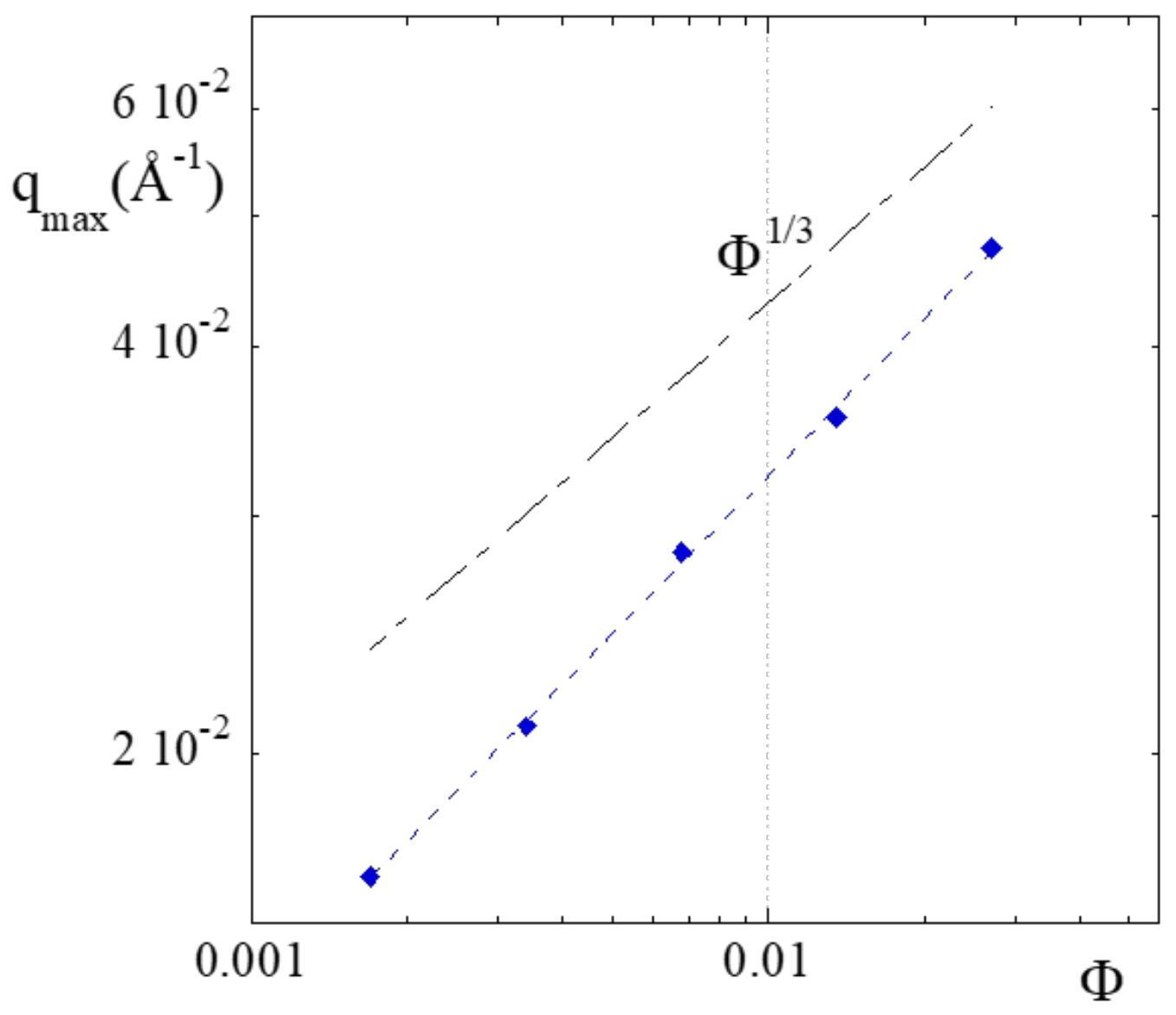

Figure 4: Dependence of the position of the structure factor peak $q_{\text {max }}$ from fig. $2 d$ in dependence on the total polymer volume fraction $\Phi$ for compound AAEt. The fit $q_{\max } \propto \Phi^{x}$ of the data points gives an exponent of $x=0.38$. The 1/3-power law is shown for comparison. 
for its Fourier transform, the structure factor [28]. This conclusion is also compatible with the characteristic lengths otained for these systems. The diameter of gyration $2 \mathrm{R}_{b l o b}$ is only slightly smaller than the smallest distance $2 \pi / q_{\max } \approx 125 \AA$ measured at the highest concentration. This is in agreement with the internal volume fraction argument discussed above: the coils are close to contact at high concentration, and increase their distance under dilution as indicated by the power law. This behaviour is consistent with the fact that more charged chains repel each other, introducing colloidal stability and resistance to aggregation.

To summarize, our SANS analysis clearly establishes that the polymers under scrutiny behave in a very different manner. While ammonioacetates form isolated coils, pyridiniocaboxylates are subdivided into blobs. These blobs must be connected, but as this is not visible in the spectra either the blob positions are totally uncorrelated or the correlation is on a length scale larger than the one probed here. Within each class of compounds, the ones with longer spacers or bulkier side groups present a stronger separation between the ammonium ion and the carboxyl group, which impedes interaction, and thus competition. PC3 and AAEt present a higher overall charge than their counterparts PC0 and AAMe which results either in the formation of smaller blobs for the pyridiniocaboxylates or repelling chains in the case of ammonioacetates.

\subsection{Polyelectrolyte multilayers (PEMs)}

According to the SANS measurements the ammonioacetate AAEt behaves like a polyelectrolyte. Former electrophoresis studies unveiled that AAEt acts like a polycation at pH 2 [12]. The consecutive dipping in polycarboxybetaine solutions of $\mathrm{pH} 2$ and solutions containing polycations like PDADMAC results in very thin layers which indicates that the surface charge was not reversed. This proves the polycation character of polycarboxybetaine at $\mathrm{pH}$ 2.

In the following the interaction of AAEt with the polyanion PSS in poly- 
electrolyte multilayers was studied and compared to the impact of its methyl counterpart AAMe.

Fig. 5 shows a linear increase in thickness with increasing number of dipping cycles. The linear fit gives a thickness increment of $45 \AA$ /double layer from

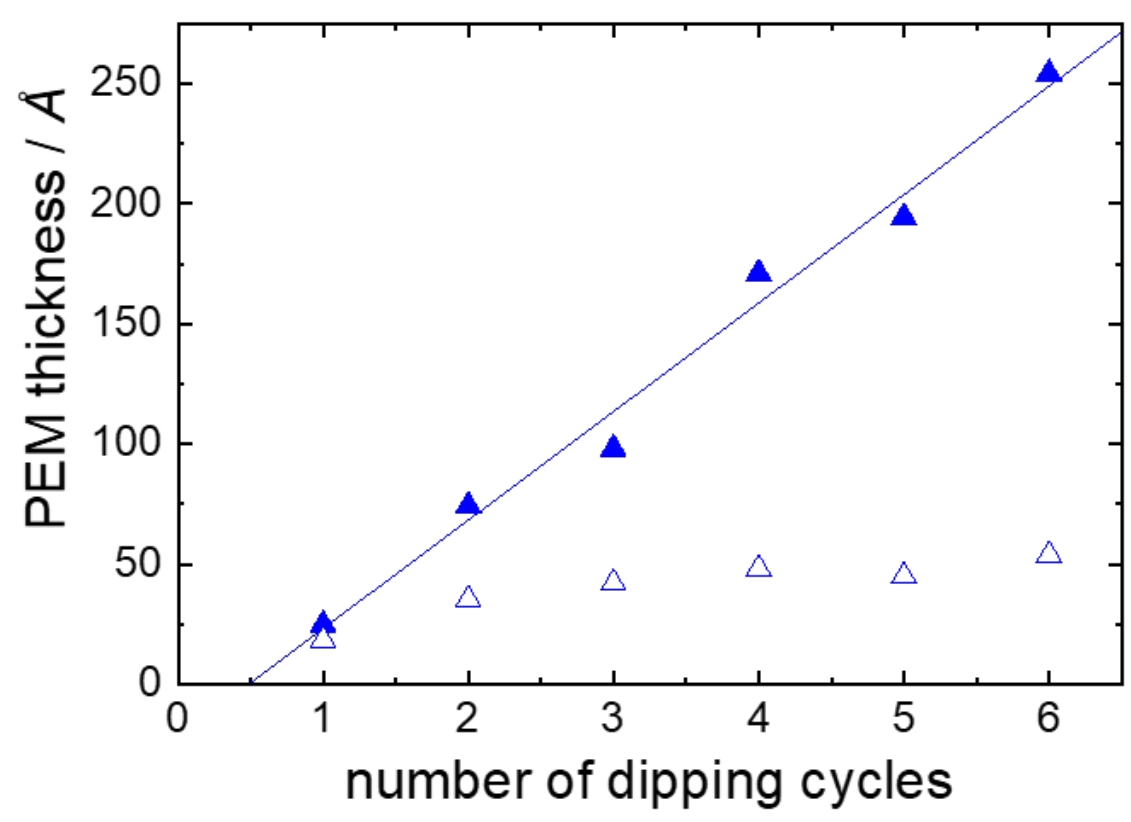

Figure 5: Multilayer thickness in dependence on the number of dipping cycles: (PSS/AAMe) ${ }_{n}$. AAMe dipping solution: $p H 2.5$ (filled symbols) and $p H$ 4.4. (open symbols). Solid line: linear fit: $45 \AA /$ double layer. PSS dipping solution: $p H=4.6$. All dipping solutions contained $\mathrm{NaCl}=0.1 \mathrm{~mol} / \mathrm{L}$.

the second double layer on. The first double layer together with the PEI layer underneath have a thickness of about $25 \AA$ all together. As expected, the first layer is thinner, since the substrate is smoother. The first layer increases the roughness and therefore the effective surface. For other systems (e.g. $\mathrm{PSS} / \mathrm{PAH}$ ) this region affected by the substrate can be extended up to three double layers [29]. The linear increase indicates a low mobility of polymer 
chains within the PEM as for e.g. PSS/PAH or PSS/PDADMAC multilayers prepared at low ionic strength. This is in contrast to PLL/HA multilayers or PEM prepared at high ionic strengths or in presence of chaotropic ions which show a growth related to a high mobility of polymer chains [30].

Fig. 6 shows the thickness of a PSS/ammonioacetate multilayer after six dipping cycles in dependence of the $\mathrm{pH}$ of the polycarboxybetaine solution.

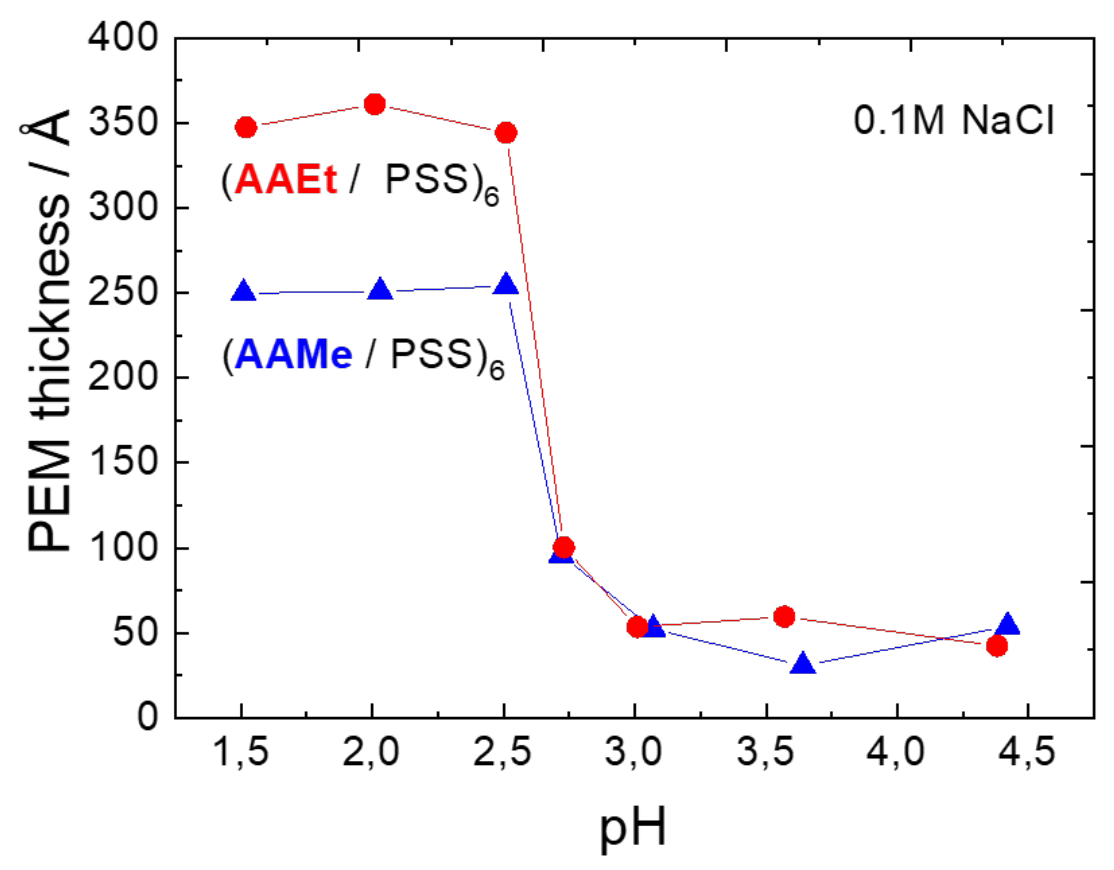

Figure 6: Thickness in dependence on the $p H$ of the polycarboxybetaine dipping solution, $\mathrm{pH}$ of the PSS dipping solutions: 4.6, 6 dipping cycles, [NaCl] $=0.1 \mathrm{~mol} / \mathrm{l}$.

Up to a $\mathrm{pH}$ of 2.5 the thickness is more or less $\mathrm{pH}$ independent and the thickness is about $250 \AA$ in the case of (PSS/AAMe) ${ }_{6}$. The (PSS/AAEt) ${ }_{6}$ multilayer is about $100 \AA$ thicker than (PSS/AAMe) 6 . Above a $\mathrm{pH}$ of 2.5 the thickness decreases abruptly to $50 \AA$ at a $\mathrm{pH}$ of 3.0 for both types of polycarboxybetaines and stays more or less constant at a thickness between 
30 and $50 \AA$ up to the $\mathrm{pH}$ of 4.5 and even to the highest studied $\mathrm{pH}$ of 9.0 (data not shown in fig. 6). The high thickness at low pH reflects the polycation character of the polycarboxybetaines due to the permanent positive charge of the nitrogen and the protonated carboxyl group. With increasing $\mathrm{pH}$ the carboxyl groups become more and more deprotonated which reduces the net charge of the polycarboxybetaine. Titration measurements [12] show a decrease in cationic charge from $80 \%$ to $50 \%$ for polycarboxybetaine AAEt and from $60 \%$ to $50 \%$ for AAMe in a pH range from 1.5 to 2.5. For most of the investigated polycation/polyanion combinations a charge density of about $50 \%$ (i.e. $50 \%$ of the monomers are charged) is sufficient for a charge reversal after each adsorption step required for multilayer formation $[31,32,33,34]$. Below a charge density of $50 \%$ for many systems the charge reversal is not sufficient anymore and the adsorption stops after a few dipping cycles [35]. Obviously, AAMe gives thinner multilayer than AAEt although the charge is similar (about 50\%) at $\mathrm{pH}$ 2.5. This is a further hint that not only the degree of charge dominates the amminioacetate chain conformation and therefore the multilayer thickness. SANS measurements mentioned above show a stronger coiling of AAEt chains than of AAMe chains (see table 2) which is in good agreement with the results for multilayers. Stronger coiling leads to thicker multilayers, as it often occurs by the addition of salt (see below).

The titrated charge of compound AAEt decreases from $50 \%$ to $30 \%$ and the one of AAMe even to $10 \%$ in a $\mathrm{pH}$ range between 2.5 and 3.0. This abrupt decrease in charge explains the sharp decrease in film thickness at $\mathrm{pH} 3.0$. The thickness of about 30 to $50 \AA$ above pH 3 in fig. 6 indicates that the adsorption stops after one to two double layers. A similar result was found for instance for the system (PSS/PDADMAC) for PDADMAC charges below $50 \%[31]$.

In addition to the low net charge above $\mathrm{pH} 3$ the configuration of the side chains might reduce the adsorbed amount. The negatively charged carboxyl groups might be turned outside towards the adsorbing PSS chain and interact 
stronger than the inner positively charged nitrogen atoms irrespective of the size of side groups at the nitrogen. This might explain why above $\mathrm{pH} 3$ the thickness is small and independent of the type of polycarboxybetaine.

As already mentioned the addition of salt during PEM preparation can have a strong effect on the polyelectrolyte chain conformation and PEM thickness. Fig. 7 shows the effect of the ionic strength of the polyelectrolyte (dipping) solutions on the thickness of multilayers.

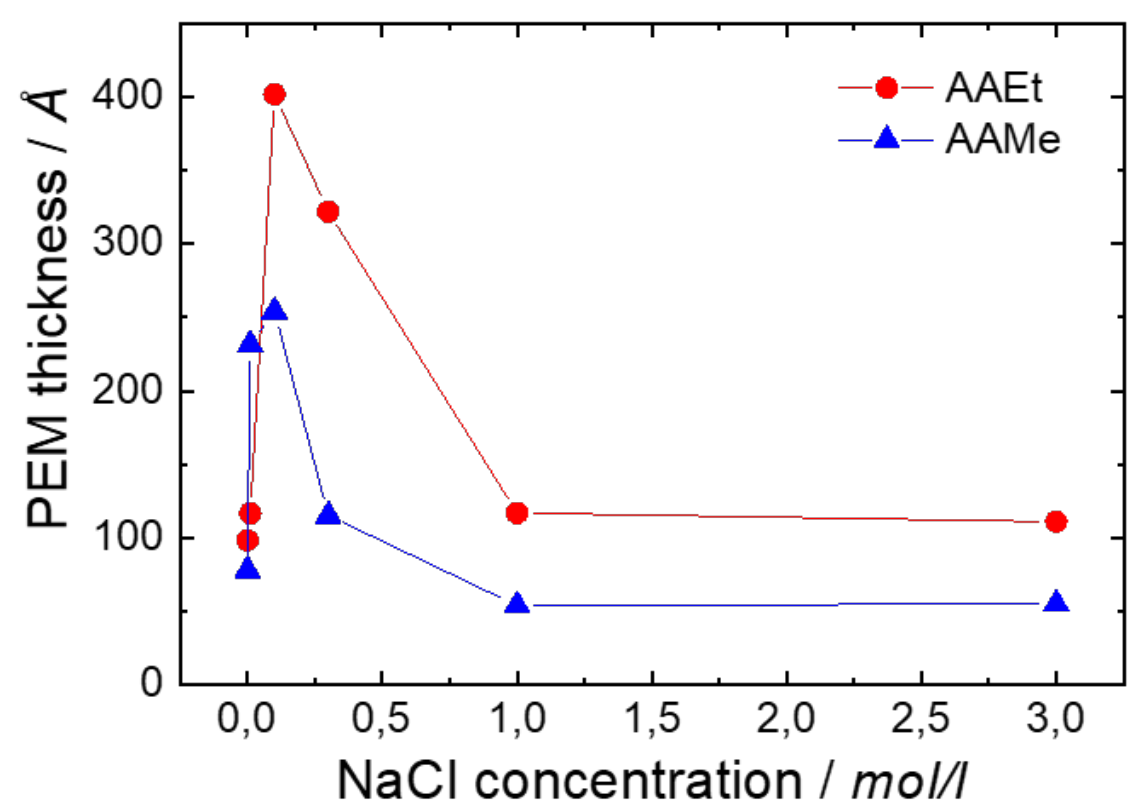

Figure 7: Multilayer thickness in dependence on the $\mathrm{NaCl}$ concentration, 6 double layers, $p H=2.5$.

Up to an ionic strength of $0.1 \mathrm{~mol} / \mathrm{L}$ the PEM thickness increases and drops down at higher salt concentrations. The thickness increase at low ionic strength can be explained by a screening of likely charged polymer segments which leads to a stronger coiling of the polyelectrolyte chains on the substrate. Furthermore, the repulsion between likely charged polyelectrolyte 
coils is screened. Both effects result in higher adsorbed amount than at lower ionic strength. At the same time, the electrostatic attraction between the adsorbing polyelectrolytes and the oppositely charged surface is screened which results in a decrease in thickness at higher ionic strengths. Therefore, a maximum in multilayer thickness occurs at a certain ionic strength $I_{\max }$. The value for $I_{\max }$ depends on the polyelectrolyte combination and can be completely different. In the present case, the maximum occurs at an ionic strength of about $0.1 \mathrm{~mol} / \mathrm{L}$. For instance for PSS/PDADMAC $I_{\max }$ is about $1 \mathrm{~mol} / \mathrm{L}$ [33]. For PSS/PAH it is above $4 \mathrm{~mol} / \mathrm{L}$, but not measurable. The thickness increases up to $4 \mathrm{~mol} / \mathrm{L}$. Above this ionic strength PSS is salted out and no homogeneous polyelectrolyte multilayers can be built up anymore. The level of $I_{\max }$ correlates with the stability of the multilayers and corresponds to the minimum outer ionic strength at which the multilayer can be destroyed after the adsorption process has been finished [36].

The given explanation is valid for "simple" polyelectrolytes with one type of charge along the chain. Obviously, it gives also the right trend for the salt experiments in the present paper. In the case of polycarboxybetaines with two types of oppositely charged groups an additional effect might become important. Due to the opposite charges, polycarboxybetaines are more coiled than "simple" polyelectrolytes. With increasing ionic strength the attraction is screened which leads to a more stretched conformation in contrast to the salt effect on the conformation of "simple" polyelectrolytes. Usually, chain stretching reduces the film thickness, which could also explain the decreasing film thickness at higher ionic strength in fig. 7 and the relatively low $\mathrm{I}_{\max }$. Hence, it is assumed that the reduced PEM thickness from $1 \mathrm{~mol} / \mathrm{L} \mathrm{NaCl}$ is due to a reduced thickness of single layers but not to the stopping of multilayer formation after a few layers. At high ionic strengths from $1 \mathrm{~mol} / \mathrm{L}$ on, the multilayer thickness is constant of about $120 \AA$ and $60 \AA$ respectively (Fig. 7). This is still thicker than the thickness of a few adsorbed layers between 30 and $50 \AA$, as shown in fig. 6 at high pH. This leads to the conclusion that at high ionic strength the formation of thin multilayers is 
still possible. It indicates that at high ionic strengths the adsorbed amount is rather affected by the intramolecular screening between the nitrogen and the carboxyl groups than by the electrostatic screening of the attraction between the adsorbing polyelectrolyte and the oppositely charged surface.

In the experiments shown in fig. 6 , only the $\mathrm{pH}$ of the polycarboxybetaines was adjusted. That means that the ratio of protonated and deprotonated carboxyl groups of the polycarboxybetaines was fixed during the PSS deposition irrespective of the $\mathrm{pH}$ during the former polycarboxybetaine adsorption step. In the experiments described in the following the $\mathrm{pH}$ of the PSS (dipping) solution was adjusted to the same value as for the respective polycarboxybetaine solution. Fig. 8 shows the thickness of the multilayer in dependence of the $\mathrm{pH}$ of PSS and polycarboxybetaine dipping solutions at an ionic strength of $0.1 \mathrm{~mol} / \mathrm{L}$. The thickness increases up to $\mathrm{pH} 2$ and decreases towards higher $\mathrm{pH}$. The maximum thickness at $\mathrm{pH} 2$ is smaller than in fig. 6 .

The $p K_{a}$ of PSS is about 3.0 [37] which explains the increase in thickness with

increasing $\mathrm{pH}$ due to an increase in PSS charge density at low $\mathrm{pH}$. Above a $\mathrm{pH}$ of 3.0 the carboxyl groups of the polycarboxybetaines become more and more deprotonated which reduces the net charge of the polycarboxybetaines. This leads to a maximum in thickness. The dissociation of PSS and the carboxyl groups are counteracting which results in a smearing out of the sharp effect in fig. 6 . The maximum thickness is smaller than in fig. 6 , since the PSS chains are not fully deprotonated in the $\mathrm{pH}$ range between 1.5 and 2.5 .

\section{Summary and conclusions}

The intermolecular interaction of polycarboxybetaines was investigated by two different experimental routes: 1) The interactions were tested in aqueous environment with SANS. The occurrence of a structure peak provided an indicator for the electrostatic repulsion between the polyelectrolytes. 2) The attraction between polycarboxybetaines and the anionic PSS was studied by 


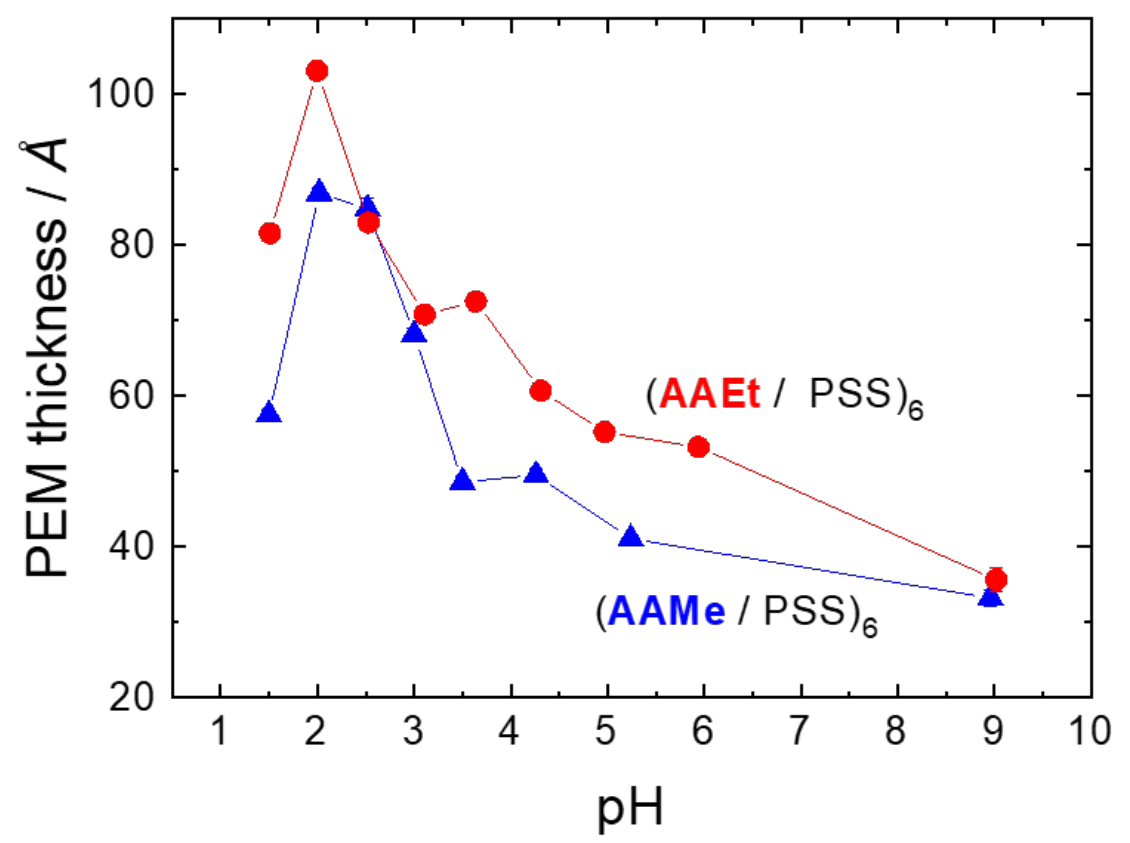

Figure 8: Thickness in dependence of the $p H$ of the PSS and polycarboxybetaine (dipping) solutions, six dipping cycles. In contrast to fig. 6 also the $p H$ of the PSS solution was changed.

the formation of polyelectrolyte multilayers from aqueous solutions. It had been known before that a system specific minimum degree of polymer charge is required for the multilayer formation.

Both types of measurement indicate that the polycarboxybetaines have polycation character at low $\mathrm{pH}$, if the intramolecular charge coupling between the quaternary nitrogen and the carboxyl group is avoided. This was reached by the chemical structure of the polycarboxybetaines. The study reveals that the sterical hinderance realized by side chains at the nitrogen is more efficient for avoiding charge coupling than a long spacer between both functionalized groups. The charge coupling seems to be independent of the polymer concentration. This leads to the conclusion that the charge coupling is mainly an 
intramolecular effect. The conformation of the polymer chains is a result of a subtle interplay between electrostatic and hydrophobic interactions including steric hinderance. This leads either to stretched pearl-necklace structures as shown for pyridiniocarboxylates ( $\mathrm{PC} 0, \mathrm{PC} 3)$ or to coil-like structures as detected for ammonioacetates (AAMe, AAEt).

\section{Acknowledgment}

First of all we would like to thank Werner Jaeger who came up with the idea to study Polycarboxybetaines long time ago. He passed away in 2011.

Julian Oberdisse, Thomas Schimmel and Regine von Klitzing acknowledge the French-German Network (supported by DFG, MPG, CEA and CNRS) and the LLB (CEA) for financing the SANS experiments at the LLB in Saclay. The two months stay of Dan F. Anghel in the group of Regine von Klitzing was financed by the SFB 448 - subproject B10 (DFG).

Our special thanks go to Gerhard Findenegg who was spokeperson of the SFB 448 "Mesoscopically structured composite systems" from 1998 to 2006 and PI of the before-mentioned French-German network, where Julian Oberdisse met him for the first time. In the early 2000's he created the idea together with Keith Gubbins from North Carolina State University (NCSU) in Raleigh to establish an international graduate school between universities in North Carolina and universities and research institutes in the Berlin/Brandenburg area which finally led to the graduate school IRTG 1524 "Self-Assembled Soft Matter Nano-Structures at Interfaces" financed by the DFG (2009 - 2017). Not only in these collaborative projects but also in daily lab-life we could experience Gerhard Findenegg as an excellent and enthusiastic scientist.

\section{References}

[1] Nierlich, M., Williams, C.; Boue, F.; Cotton, J.P.; Daoud, M.; Farnoux, B.; Jannink, G.; Picot, C.; Moan, M.; Wolff, C.; Rinaudo, M.; de 
Gennes, P.G.: J. Physique 40 (1979) 701.

[2] Nishida, K.; Kaji, K.; Kanaya, T.: Macromolecules 28 (1995) 2472.

[3] Förster, S.; Schmidt, M.: Adv. Polym. Sci. 120 (1995) 51.

[4] Essafi, W.; Lafuma, F.; Wiliams, C.E.: Eur. Phys. J. B 9 (1999) 261.

[5] Claesson, P.M.; Bergström, M.; Dedinaite, A.; Kjellin, M.; Legrand, J.-F.; Grillo, I.: J. Phys. Chem. B 104 (2000) 11689.

[6] de Gennes, P.-G.; Pincus, P.; Velasco, R. M.; Brochard, F.: J. Phys. France 37 (1976) 37.

[7] Odijk, T.: J. Polym. Sci., Polym. Phys. Ed. 15 (1977) 688.

[8] Skolnick, J.; Fixman, M.: Macromolecules 10 (1977) 944.

[9] Dobrynin, A.V.; Colby, R.V.; Rubinstein, M.: Macromolecules 28 (1995) 1859.

[10] Barrat, J.L.; Joanny, J.F.: Adv. Chem. Phys. 94 (1996) 1.

[11] E. Schneck, D. Horinek, R. R. Netz, J.Phys.Chem.B 2013, 117, 8310.

[12] J. Bohrisch, T. Schimmel, H. Engelhardt, W. Jaeger, Macromoelcules 35 (2002) 4143.

[13] C. Üzüm, S. Christau, R. von Klitzing Macromolecules 44 (2011) 77827791.

[14] R. von Klitzing, B. Kolaric, W. Jaeger, A. Brandt Phys. Chem. Chem. Phys., 2002 4, 1907.

[15] G. Decher, J.D. Hong, J. Schmitt: Thin Solid Films 210/211 (1992) 831.

[16] R.M.A. Azzam and N.M. Bashara, in Ellipsometry and Polarized Light, Elsevier, Amsterdam (1987) 
[17] H. Motschmann and R. Teppner, Ellipsometry in Interface Science, in Novel Methods to study Interfacial Layers, eds. D. Möbius, R. Miller, Studies in Interface Science 11 Elsevier, Amsterdam (2001)

[18] P. Schurtenberger in Neutrons, X-ray and Light Scattering P. Lindner, T. Zemb; eds.; North Holland, 2002

[19] R. Schweins, K. Huber Macromol. Symp. 2004, 211, 25.

[20] S. Lages, G. Goerigk, K. Huber Macromolecules 2013, 46, 3570.

[21] P. Pusey, in Neutrons, X-ray and Light Scattering P. Lindner, T. Zemb; eds.; North Holland, 2002

[22] S. B. Mahmoud, W. Essafi, A. Brulet, F. Boue Macromolecules 2018, 51, 9259 .

[23] Y. Zeng, S. Grandner, C.L.P. Oliveira, A.F. Thünemann, O. Paris, J.S. Pedersen, S.H.L. Klapp, R. von Klitzing Soft Matter 7201110899.

[24] M. Ludwig, R. v. Klitzing, Adv. Coll. Interf. Sci. 2019, 269, 270.

[25] S. H. Chen, E. Y. Sheu, J. Kalus, H. Hoffman J. Appl. Cryst. 1988, 21, 751.

[26] J. I. Hayter, J. Penfold J. Mol. Phys. 1981 42, 109.

[27] J. P. Hansen, J. B. Hayter Molecular Physics 198246651.

[28] R. Klein, in Neutrons, X-ray and Light Scattering P. Lindner, T. Zemb; eds.; North Holland, 2002

[29] M. Lösche, J. Schmitt, G. Decher, W.G. Bouwman. K. Kjaer: Macromolecules 31, 8893 (1998)

[30] D. Volodkin, R. von Klitzing (2014) Curr. Op. Coll. Interf. Sci. 1925.

[31] R. Steitz, W. Jaeger, R.v.Klitzing: Langmuir 17, 4471-4474 (2001). 
[32] Glinel, K.; Moussa, A.; Jonas, A. M.; Laschewsky, A.; Langmuir; (Article); 2002, 1408-1412.

[33] B. Schoeler, G. Kumaraswamy, F. Caruso Macromolecules 35 (2002) 889.

[34] U. Voigt, W. Jaeger, G. H. Findenegg, R. v.Klitzing J. Phys. Chem. B. 107 (2003) 5273.

[35] U. Voigt, V. Khrenov, K. Tauer, M. Hahn, W. Jaeger, R. v. Klitzing J. Phys: Condens. Matter. 15 (2003) S213.

[36] P. Nazaran, J. E. Wong, R. v. Klitzing, non-published results.

[37] M. Mandel, Polyelectrolytes. In: Encyclopedia of Polymer Science and Engineering, 2nd edition, Eds.: F.H. Mark, N.M. Bikales, C.G. Overberger, G. Menges, Wiley, New York, 1988, 11, 739. 